\title{
Auszeichnung
}

\section{Jürgen M. Steinacker erhält Hufeland-Preis 2014}

Für das erfolgreiche Präventionsprogramm „Komm mit in das gesunde Boot“ ist Professor Dr. Jürgen M. Steinacker, Ärztlicher Leiter der Sektion Sport- und Rehabilitationsmedizin des Universitätsklinikums Ulm, mit dem Hufeland-Preis 2014 ausgezeichnet worden. „Wir möchten mit dem Programm „Komm in das gesunde Boot" den Kindern zeigen, wie viel Spaß Bewegung und gesundes Leben machen", sagte Steinacker bei der Preisverleihung. Der von der Deutschen Ärzteversicherung gestiftete und von der Bundesärztekammer und der Bundeszahnärztekammer sowie von der Bundesvereinigung Prävention und Gesundheitsförderung mitgetragene Preis hat das Ziel, die Präventivmedizin in Deutschland zu fördern. Für den Hufeland-Preis 2015 läuft derzeit das Auswahlverfahren, um den Hufelandpreis 2016 können sich Ärzte und Zahnärzte allerdings noch bis zum
31. Oktober 2016 bewerben. Die Ausschreibungsunterlagen sind unter www.hufeland-preis.de hinterlegt. Mit dem HufelandPreis werden jedes Jahr Arbeiten auf dem Gebiet der Gesundheitsvorsorge oder präventionsbezogenen Versorgungsforschung prämiert. Der Preis ist mit 20.000 Euro dotiert. red

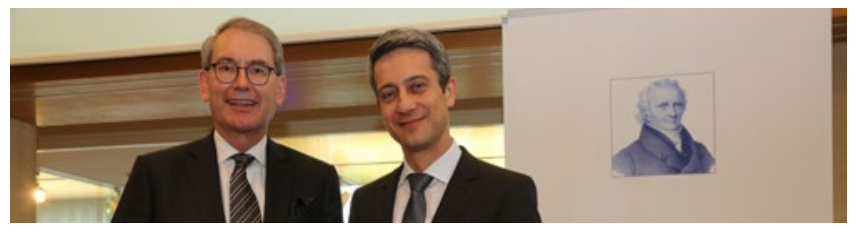

Professor Dr. Jürgen M. Steinacker (li.) bei der Preisübergabe durch Timmy Klebb, Vorstandsvorsitzender der Deutschen Ärzteversicherung

Preisverleihung

\section{Präventionspreis Frühkindliche Karies}

Gleich vier Preisträger hat die Bundeszahnärztekammer (BZ̈̈K) in Kooperation mit dem Hersteller von Mund- und Zahnpflegeprodukten CP GABA mit dem „Präventionspreis Frühkindliche Karies“ beim Deutschen Zahnärztetag in Frankfurt ausgezeichnet. Den Preis, der mit insgesamt 5000 Euro dotiert war, hatte die „Initiative für eine mundgesunde Zukunft in Deutschland“ ausgeschrieben. Der erste Platz ging an eine Arbeitsgruppe um Prof. Dr. Hüsamettin Günay von der Medizinischen Hochschule Hannover. Das Konzept der „Zahnärztlichen Gesundheitsfrühförderung interdisziplinär“ begleitet werdende Mütter und ihre Kinder bereits in der Schwangerschaft. Die Urkunde für den zweiten Platz nahm Dr. Michael Schäfer vom Gesundheitsamt Düsseldorf entgegen. Das Präventionsprogramm „Zukunft für Kinder in Düsseldorf“ bietet koordi- nierte Hilfen für Eltern und Kinder im Alter bis drei Jahre und richtet sich an Risikofamilien. Mit dem Ernährungsprogramm „Kita mit Biss“ belegte der Verein Gesundheit Berlin-Brandenburg den dritten Platz. Mit einem Sonderpreis ehrte die Jury die Arbeit der Stiftung Pro Kind, die mit einem Hausbesuchsprogramm bei Erstgebärenden im zweiten Drittel der Schwangerschaft Prävention betreibt.

Die Jury aus vier Experten achtete bei der Auswahl der Gewinner darauf, dass die Konzepte und Projekte wissenschaftlich fundiert, bundesweit umsetzbar und dazu geeignet sind, Eltern, Erziehungsberechtigte und Betreuer für ein verbessertes Ernährungs- und Mundhygieneverhalten zu sensibilisieren und zur Umsetzung zu motivieren.

red

\section{Wrigley-Prophylaxe-Preis}

\section{Erster Platz für Studie zu Auswirkungen von Crystal Meth}

Der erste Platz des Wrigley-Prophylaxe-Preises ist in diesem Jahr an ein Münchener Studienteam gegangen, das sich mit den verheerenden Auswirkungen der Szenedroge Crystal Meth auf die Zahngesundheit beschäftigt hat. Die Vergleichsstudie des Autorenteams um Dr. Dr. Niklas Rommel an 200 Patienten mit und ohne Drogenkonsum liefert erstmals umfangreiche Daten zum Zahnschädigungspotenzial von Crystal Meth: Die abhängigen Patienten hatten signifikant häufiger Karies, Gingivitis, Parodontitis und Zahnhartsubstanzverluste, eine schlechtere Mundhygiene, eine reduzierte Speichelfließrate und -pufferkapazität. Hauptursache dafür ist wahrscheinlich der sympathomimetische Effekt der Substanz mit resultierender Mundtrockenheit und Zähneknirschen, fand das Forscherteam heraus. Brennpunkt sind vor allem die Grenzregionen zu Tschechien, da die dortige liberale Drogengesetzgebung seit $2010 \mathrm{zu}$ einem massiven Anstieg des Konsums auch bei deutschen Jugendlichen geführt hat.

Den zweiten Platz belegt eine Untersuchung aus Dresden zum erosionsprotektiven Potenzial von Pflanzenextrakten. Den Sonderpreis teilen sich ein Prophylaxeprojekt für Grundschulkinder in Wuppertal und eine Kooperationsinitiative zwischen Zahn- sowie Kinder- und Jugendärzten in Mönchengladbach.

Der Wrigley-Prophylaxe-Preis zeichnet seit 22 Jahren herausragende Arbeiten auf dem Gebiet der Kariesprophylaxe aus und ist mit insgesamt 12.000 Euro dotiert. Er fördert erfolgreiche Präventionsinitiativen, rückt aber auch Probleme mit dringendem Handlungsbedarf in den Fokus der Öffentlichkeit. 